\title{
Brain SubStratum of PATHOGENiC NeURotic Belief in Panic Disorder: A Single Case STUd Y
}

\author{
Miloslav Kukleta * \\ Department of Psychiatry, First Medical Faculty, Charles University, Prague, \\ Central European Institute of Technology (CEITEC), Masaryk University, Brno, \\ Czech Republic
}

\begin{abstract}
The aim of the study was to demonstrate how recent research achievements in the neuroscience of emotions can influence practical management of anxiety disorders. Using the data obtained from analysis of psychophysiological mechanisms in one case of panic disorder, the type of threat, key threat stimuli, and the structure of the pathogenic neurotic belief were ascertained and, on this basis, an individually tailored therapeutic procedure was designed. Its application resulted in almost complete dissipation of the neurotic belief, which represented the key pathogenic mechanism in the case studied. The study called attention especially to the importance of detailed diagnostics of memory mechanisms underlying the formation of neurotic belief in the psychotherapy of anxiety disorders.
\end{abstract}

Key w ord s: cognitive behavioral therapy; neurotic belief; associative learning; panic disorder

\section{INTRODUCTION}

The detection of a threatening event and the control of defense against it are brain capabilities of basic survival value. Recent advances in animal and human research elucidated the organization principles of systems underlying these processes, their neuronal circuitry and molecular mechanisms that provide new insights into normal as well as pathological executive processes related to these important behavioral functions. Key neural circuits of the system present limbic structures including amygdala and insula, as well as other interconnected structures such as the periaqueductal gray and hypothalamus (LeDoux, Cicchetti, Xagoraris \& Romanski, 1990; LeDoux, 1995; Armony \& LeDoux, 2000; Phan, Wager, Taylor \& Liberzon, 2002; Kober, Barret, Joseph, Bliss-Morreau, Linquist \& Wager, 2008). All these structures interact with a number of cortical areas involved in cognitive functions but the system may act relatively independently, without conscious recognition of a threatening stimulus (Öhman, Flykt \& Lundqvist, 2000; Öhman, 2005).

A basic step of adaptive behavior and normal functioning represents recognition of threat stimuli based on both innate and learned experiences. While innate stimuli are limited in number, the acquired ones result from remembering of various cues associated with danger experiences in the previous life. When a danger is recognized, the system triggers a defense response which is partly innate, "pre-programmed" and partly individually specific. The response includes autonomic and endocrine changes, more or less specific behaviors and

*Correspondence to: Miloslav Kukleta, Central European Institute of Technology (CEITEC), Masaryk University, Brno, Kamenice 126/3, CZ-62500 Brno, Czech Republic, e-mail: mkukleta@med.muni.cz, Tel: 00420549495117 Received July 11, 2011; accepted July 27, 2011; Act Nerv Super (Praha) 53(3-4),159-65. 
accompanying feelings of fear or anxiety. Whereas fear is a response to an actual danger and is typically triggered by specific stimuli, anxiety is mostly considered as a state of preparation for a predicted threat, which can be real or imaginary.

Current functional neuroimaging studies focused on clinically significant anxiety accumulated great amount data for a quantitative meta-analysis. Etkin and Wager (2007) have recently published such analysis for emotional processing in posttraumatic stress disorder (PTSD), social anxiety disorder (SAD), and specific phobia. According to these findings common conditions related to these disorders were greater activation of the amygdala and insula in patients, compared to matched controls. Besides this characteristic shared across disorders, there were also important differences between them. Most striking was the marked hypoactivation in portions of the cingulate and prefrontal cortices in PTSD patients, which was not observed in SAD and specific phobia (Etkin \& Wager, 2007).

Although anxiety disorders commonly involve an innate mechanism that unfolds during life, such as a tendency for extreme shyness, various other mechanisms and functions contribute significantly to anxiety pathologies (Barlow, 2000). In the pathogenesis of panic disorder there are at least two such contributing mechanisms: the Pavlovian fear conditioning, which increases a number of threat stimuli, and formation of neurotic belief, which could explain amplification of the perceived threat and the self-perpetuating character of the disorder.

In this context, the present clinical practice stresses the importance of two aspects of brain functioning in anxiety disorder: (i) frequent occurrence of maladaptive patterns of thinking and (ii) formation of beliefs that underlie such thinking. The remarkable efficiency of cognitive-behavioral treatment of these disorders that focuses on thoughts and beliefs justifies this recognition of the pathogenic role of the factors mentioned (Barski \& Ahern, 2004; Barlow, Gorman, Shear \& Woods, 2004). Based on these findings an intention of the current study is to demonstrate one selected case of panic disorder as an example how the present understanding of defense mechanisms may be useful in practice of psychotherapy.

The main aim of the study was to demonstrate that the recurrent perception of threat to life was in this patient the result of misinterpretation of one or two key symptoms by his neurotic belief. Using clinical data and data from testing the emotiogenity of the selected stimuli, the disorder was analyzed in terms of the threatening stimuli, of the character of the experienced threat, of the mechanisms which transformed numerous insignificant or benign stimuli into threatening ones, and of the mechanisms that contributed to the long-lasting duration of this transformation. The creation and application of an individually shaped psychotherapeutic procedure, which was designed to modify his neurotic belief, was the second aim of the study.

\section{METHODS}

The patient (a married man, aged 48 years, employed as a car driver) started individual cognitive behavioral (CB) intervention in the course of his hospitalization in a psychiatric clinic where he was admitted with a severe form of panic disorder (F 41.0 according to DSM IV). His first contact with psychiatry shortly preceded the hospitalization. His neurotic troubles were for many years disregarded and interpreted by specialists as an unimportant corollary of cervicobrachial pathology and dysphagia of unknown origin. At the time of hospitalization his inability to work lasted for almost one year.

The gathering of relevant data and therapeutic intervention were done in sessions of varying duration (more than $60 \mathrm{~min}$ at the beginning, approximately $30 \mathrm{~min}$ at the end of intervention), which took place at varying intervals (two sessions during a week at the beginning, one session per two weeks at the end). The sessions were structured according to the diagnostic and therapeutic progress. The major tasks of the CB intervention could be listed as follows: (1) ascertaining the key anxiogenic symptom; (2) ascertaining the level of conviction that this symptom was the sign of a major threat to life; (3) looking for a procedure 
for intentional induction of this symptom, and, by this way, of threat states; (4) detailed explanation of anatomic and physiologic knowledge relevant to the disorder (breathing; function of the brain defense system); (5) exploration of the patient's neurotic experiences with the aim to find out confirmation of expert explanations of what is behind his anxiety disorder; (6) demonstration that the key symptom and, consequently, the anxiety state can be intentionally evoked and removed; (7) formulation of rational arguments against wrong interpretation of the significance of the key symptom; (8) elaboration of an efficient nonpharmacological procedure which the patient could use for mastering his anxiety states issued from everyday situations.

In the therapeutic work directed interviews were used as a basic tool for gathering relevant data, as for instance history of anxiety troubles, hypochondriac attitudes and beliefs, role impairment, changes in functional status, medical co-morbidity, etc. The assessment of the anxiety states that occurred during therapeutic sessions was based on the patient's detailed description of evoked feelings and on observation of visible behavioral changes. The intensity of the states was expressed by the patient as a comparison with the strongest analogous state experienced in the past. The measure of conviction, with which the patient held his wrong belief was based on its evaluation on a graphical scale. The present state of neurotic psychopathology was assessed by Knobloch's Inventory of neurotic symptoms N 5, which gathers data about the prevalence and intensity of 33 frequent neurotic symptoms. The anxiety states induced during the sessions were removed by a procedure based on a progressive muscle relaxation technique. The attempt to correct neurotic belief started from the first session and the procedure used in it evolved in the course of the intervention according to the information obtained in a parallel diagnostic investigation. Its basic idea was to offer the patient sufficient factual and experiential material for an attitude change. In the latter case it comprised material from hierarchically different brain mechanisms supposedly engaged in the formation and maintenance of the belief.

\section{RESULTS}

\subsection{Type of threat}

From basic threat conditioning situations (threat to somatic or mental integrity, threat to social status, and threat of major loss) the threat to life by constriction of airways was determined as a dominant recurring subjectively perceived menace. The perception of the threat was not the chief complaint; nevertheless, when directly asked, the patient realized this aspect of his health problems without great hesitation. The level of threat fluctuated between high during anxiety states and low in periods between attacks.

\subsection{Key threat stimuli}

The dyspnea linked with a feeling of pressure on the upper sternum was established as the key symptom. The patient believed that the dyspnea that was associated with the symptom could cause suffocation and death. Every anxiety state that evolved during therapeutic sessions was preceded by this symptom. Moreover, the whole set of subjective feelings and specific behaviors of anxiety states comprised tachypnea, impaired swallowing, feeling of tension, dizziness, inner trembling, sweating, palpitations, impaired seeing, restlessness, visible attempts to swallow, and rapid eyelid movements. The anxiety states occurring during the sessions (35 states) evolved either spontaneously in the context of the patient's reports about past significant life situations or issued from the therapist's attempts to induce them intentionally. 


\subsection{Intentional evocation of threat states}

At first diagnostic and soon afterwards therapeutic reasons led us to intentional evocation of anxiety states. The states were induced by imagining past situations in which anxiety states evolved (4 cases) or by various maneuvers, such as mild physical load, Rhomberg posture, orthostatic response, apnea, hyperventilation, and increase of intrapulmonary pressure (27 cases). The latter procedure was finally selected for therapeutic application of evoked anxiety (20 cases). The increase of intrapulmonary pressure reliably induced the key symptom until the last session in which intentionally evoked anxiety was used (session No. 13). The intensity of induced anxiety remained approximately at $50 \%$ of its maximal possible value all over this period.

\subsection{Structure of the pathogenic belief}

The brain substratum of the pathogenic belief could be imagined as a nonspecific evaluation system enabling transfer of information and its comparison with memory and several distinct memory records related to the neurosis. At the beginning of CB intervention the patient used the following chain of arguments for explaining the perceived menace to life: pressure on upper chest signals constriction of the upper airways leading to insufficient lung ventilation which could cause suffocation (conclusion further supported by the accompanying dyspnea). After accepting factual correction of this mistaken cognitive evaluation (in the 5th session), the patient explained his persisting high conviction about the life-threatening character of his attacks by stating that he just feels it so. These two reports suggest implication of at least two memory systems in the structure of the patient's neurotic belief - memory for facts and associative memory. As revealed by the analysis of the patient's medical history, the association between pressure on chest or dyspnea, and the state of threat to life could take place in the past during an episode of his first intense anxiety state, which was considered as a heart attack. This association was strengthened many times after this event during the subsequent neurotic development and represented a more important component of the belief because of its biological deep roots. It is conceivable that a cognitive explanation of the symptom significance appeared second arily.

\subsection{Procedure for correcting the pathogenic belief}

The main demonstrations and arguments offered to the patient for correcting his interpretation of the key symptom were as follows: (1) the upper airways remain open even in the anxiety state; (2) the control of breathing is basically an automatic process; (3) respiratory muscles are under voluntary control even in anxiety; (4) the key symptom and consecutive anxiety can be intentionally induced and removed; (5) reorientation of attention can modify the intensity of anxiety; (6) new anxiogenic stimuli and situations can be created by fear conditioning; (7) none of the hundreds of anxiety states experienced in the past compromised the patient's life; (8) the therapist whose basic professional imperative is not to harm his patient induced intentionally these "highly dangerous states"; (9) demonstration of the defense system role in a misinterpretation of the key symptom and in other aspects of the anxiety disorder. Development of the patient's conviction with which he held the belief that dyspnea was a life-threatening state is documented in Table 1. 
Table 1. Patient's estimation of the level of conviction with which he held the belief that the prominent anxiety symptoms represented for him a major threat to life and other relevant data from CB intervention.

\begin{tabular}{|l|l|l|l|l|l|} 
Session & Day of CBT & Conviction & Symptoms & Status & Extra anxiolytics \\
1 & $1^{\text {st }}$ & $96 \%$ & $24 / 37$ & $\mathrm{H}$ & \\
2 & $6^{\text {th }}$ & $96 \%$ & & $\mathrm{H}$ & \\
3 & $8^{\text {th }}$ & $96 \%$ & & $\mathrm{H}$ & \\
4 & $13^{\text {th }}$ & $96 \%$ & & $\mathrm{H}$ & \\
5 & $15^{\text {th }}$ & $96 \%$ & & $\mathrm{H}$ & \\
6 & $20^{\text {th }}$ & $89 \%$ & & $\mathrm{H}$ & \\
7 & $22^{\text {nd }}$ & $86 \%$ & & $\mathrm{H}$ & \\
8 & $27^{\text {th }}$ & $73 \%$ & & OC & Used \\
9 & $31^{\text {st }}$ & $70 \%$ & $24 / 38.5$ & OC & Used \\
10 & $36^{\text {th }}$ & $71 \%$ & & OC & Used \\
10 & $36^{\text {th }}$ & $41 \%$ & & OC & Used \\
11 & $41^{\text {st }}$ & $39 \%$ & & OC & 4 tbl \\
12 & $48^{\text {th }}$ & $9 \%$ & & OC & 4 tbl \\
13 & $57^{\text {th }}$ & $7 \%$ & $18 / 20$ & OC & 2 tbl \\
14 & $71^{\text {st }}$ & $5 \%$ & $17 / 17$ & OC & 0 tbl \\
15 & 84 th & $2 \%$ & $16 / 16$ & OC & 1 tbl \\
\hline
\end{tabular}

Note: CBT - cognitive behavior therapy; Symptoms - maximal number of symptoms 33, maximal intensity of symptoms 99 , i.e. 33/ 99; $\mathrm{H}$ - hospitalization, OC - outpatient care

Further data presented in Table 1 demonstrate a considerable relief from neurotic symptoms at the end of intervention and an increased ability to master everyday anxiety states by non-pharmacological procedure. Until the last session the patient reported frequent occurrence of such states; however, contrary to the period before correction of his neurotic belief these states were of short duration, and though qualified as disagreeable, they were not considered as life-threatening. The patient was able to stop their development within a period of tens of seconds by a special behavioral procedure which issued from CB intervention. In fact he used his own procedures for mastering anxiety states even in the past, prior to the intervention, but their efficacy was incomparably lower. Another noticeable change which evolved after correction of the patient's neurotic belief was his increased ability to follow detailed genesis of anxiety states. He repeatedly reported on new, so far unidentified anxiogenic stimuli and situations.

\section{DISCUSSION}

Much of our present understanding of the brain mechanisms involved in the human anxiety comes from studies of fear conditioning in animals. The study of neurobiologic basis of fear and anxiety started at the beginning of the 20th century and its course can be marked by several major developments (Cannon, 1929; Papez, 1937; Klüver \& Bucy, 1939; MacLean, 1952). This research accumulated data which finally made it possible to draw the brain system mediating the defense against threat (Aggleton \& Mishkin, 1986; LeDoux, 1987). The application of this animal model for the explanation of human emotions in the field of neurotic anxiety have brought better understanding of the pathogenic mechanisms of these disturbances and very efficient therapeutic procedure - the cognitive behavioral therapy.

In the context of defense system functioning the current study concerns the durability of the fearful experience. Its main result was the demonstration of an almost complete dissipation of neurotic belief, which represented the key pathogenic mechanism in the case of panic disorder studied. This result was obtained in spite of the fact that the emotiogenity of 
key threat stimuli remained intact. The finding is consistent with observations about the frequent return of anxiety after successful treatment in human (Rachman, 1989) and about the reinstatement of fear responses following successful extinction after placing the animal into a different context (Bouton \& Swartzentruber, 1991; Jacobs \& Nadel, 1985) and suggests that overcoming fear involves acquiring completely new memories. Thus, in our case the patient learned in some new context that the key stimuli no longer signal threat to live.

The more general contribution of the study was the demonstration how recent research achievements in the neuroscience of defense behavior could influence practical managem ent of anxiety disorders. The opinion that neurotic anxiety is in the majority of cases an adequate response to a threat could even have a breakthrough character in this clinical domain. In the diagnostics of these disorders attention should be focused on the stimuli or situations which the patient evaluates as threatening, on circumstances of their formation and long-lasting persistence, on the character and duration of induced threat states, and on mechanisms which are able to make other efficient threat stimuli from previously neutral or benign ones. In psychotherapy this new understanding would enable to formulate goals directed to key pathogenic mechanisms, such as misinterpretation of the significance of definite stimuli, etc.

In the light of present knowledge about the function of the defense system, the story of our patient could be retold in the following way. His neurotic troubles started in the past by an event whose memories were very vivid and reports on it surprisingly detailed in spite of the fact that it happened 14 years ago. At that time, in the context of a long-lasting working overload, the patient experienced an intense state of threat, which comprised severe dyspnea, feeling of suffocation, feeling of airways constriction, signs of hypocapnea, and intense fear of life because he considered the state as a manifestation of myocardial infarction or some other life-compromising event. Though any detailed analysis of the resulting memory record was not possible due to the time elapsed, some id ea about its structure could be formulated. Similarly as the memory from fear learning in animal experiments (Kukleta, 1972), which was shown to consist of separate components, the record of the event had to have multiple brain representation. The participation of a declarative memory system for the events was certain as was the participation of a system for learned facts. The former created a temporarily categorized record of the whole event, the latter enabled evaluation of the health risk of the situation. Another memory component was created by the mechanism of emotional associative learning, which linked various sensory aspects of the event with threat to life. Thorough clinical examination which followed the event excluded any serious pathology and this conclusion influenced the memory components, but not in the same way. New, more recent information about medical examinations was added to the register for events and factual memory was updated by new data about his health state. Contrary to these favorable modifications of cognitive memory components the malignant significance of bodily stimuli, which was obtained by associative learning, remained untouched. These associations evidently became the core of future neurotic belief in which the symptoms interpreted as signs of possible suffocation (pressure on upper chest, dyspnea) played a leading role. Sporadic anxiety states happening in the following years prevented extinction of these associations and contributed to further neurotic development. Five years before the intervention, short-lasting, transitory constrictions of the upper esophagus enlarged the set of anxiety symptoms. This functional disturbance of esophageal peristalsis transformed eating into a life-threatening activity with perceived threat dependent on the water content of the swallowed morsel.

The formulation of the chain of causes and consequences in the neurotic development of our patient was based on verifiable data from the patient's medical history, on testing the emotiogenity of the stimuli selected, and on the knowledge of brain memory mechanisms. In conclusion, the study attempted to point out some reserves in the diagnostics of mechanisms implicated in individual neurotic development and to show how focusing on these mechanisms can serve in the designing of efficient cognitive behavioral therapeutic procedures. 


\section{ACKNOWLED GEMEN T}

The author thanks for support by grants MSM 0021620849 and MSM 0021622404.

\section{REFEREN CES}

Aggleton, J.P. \& Mishkin, M. (1986). The amygdala: sensory gatew ay to emotions. In R. Pluchik \& H. Kellerman (Eds), Emotion: Theory, Research and Experience, Volume III Biological Foundation of Emotion, Academic Press, New York, pp. 281-299.

Armony, J.L. \& LeDoux, J.E. (2000). How danger is encoded: tow ard a systems, cellular, and computational understanding of cognitive-emotional interactions in fear. In M.S. Gazzaniga (Editorin-chief), The new cognitive neurosciences. A Bradford book, The MIT Press, Cambridge, Massachusetts, London, England, pp. 1067-1080.

Barlow, D.H. (2000). Unraveling the mysteries of anxiety and its disorders from the perspective of emotion theory. American Psychologist 55, 1247-1263.

Barlow, D.H., Gorman, J.M., Shear, M.K. \& Woods, S.W. (2000). Cognitive-Behavioral Therapy, Imipramine, or Their Combination for Panic Disorder. JAMA 283, 2529-2536.

Barski, A.J., Ahern, D.K. (2004). Cognitive behavior therapy for hypochondriasis. JA MA 291, 1464-1470.

Bouton, M.E. \& Swartzentruber T. (1991). Sources of relapse after extinction in Pavlovian and instrumental learning. Clinical Psychology Review 11, 123-140.

Cannon, W.B. (1929). Bodily changes in pain, hunger, fear and rage (2nd ed.). New York, Appelton.

Etkin, A. \& Wager, T.D. (2007). Functional neuroimaging of anxiety: a meta-analysis of emotional processing in PTSD, social anxiety disorder, and specific phobia. American Journal of Psychiatry 164, 1476-1488

Jacobs, W.J. \& Nadel, L. (1985). Stress-induced recovery of fear and phobias. Psychological Review 92, $512-531$

Klüver, H. \& Bucy P.C. (1939). Preliminary analysis of functions of the temporal lobes in monkeys. Archives of Neurology and Psychiatry 42, 979-1000.

Kober, H., Barrett, L.F., Joseph, J., Bliss-Moreau, E., Linquist, K. \& Wager, T.D. (2008). Functional grouping and cortical-subcortical interactions in emotion: a meta-analysis of neuroimaging studies. Neuroimage 42, 998-1031.

Kukleta, M. (1972). Heterogeneity of memory components of the skilled escape reaction in the rat. TIT Journal of Life Sciences 2, 53-62.

LeDoux, J.E. (1987). Emotion. In: F. Plum, V.B. Mountcastle, American Physiological Society \& S.R Geiger (Eds), Handbook of Physiology, Nervous system V, Higher functions, pp. 350-398.

LeDoux, J.E. (1995). Emotion: clues from the brain. Annual Review of Psychology 46, 209-235.

LeDoux, J.E., Cicchetti, P., Xagoraris, A., Romanski, L.M. (1990). The lateral amygdaloid nucleus: sensory interface of the amygdala in fear conditioning. Journal of Neuroscience 10, 1043-1054.

MacLean, P.D. (1952). Some psychiatric implications of physiological studies on frontotemporal portions of limbic system (visceral brain). Electroencephalography and Clinical Neurophysiology 4, 407418.

Öhman, A., Flykt, A., Lundqvist, D. (2000). Unconscious emotions: evolutionary perspectives, psychophysiological data, and neuropsychological mechanisms. In R.D. Lane \& L. Nadel (Eds), Cognitive neuroscience of emotion. Oxford University Press, New York 2000, pp. 296-327.

Öhman, A. (2005). The role of the amygdala in human fear: automatic detection of threat. Psychoneuroendocrinology 30, 953-958.

Papez J.W. (1937). A proposed mechanism of emotion. Archives of Neurology and Psychiatry 79, 217-224.

Phan, K.L., Wager, T., Taylor, S.F. \& Liberzon, I. (2002). Functional neuroanatomy of emotion: a metaanalysis of emotion activation studies in PET and fMRI. Neuroimage 16, 331-348.

Rachman, S. (1989). The return of fear - review and prospect. Clinical Psychology Review 9, 147-168.

Rathgeb-Fuertsch, M., Kempter, G., Feil, A., Pollmächer T., Schuld, A. (2011). Short- and long-term efficacy of cognitive behavioral therapy for DSM-IV panic disorder in patients with and without severe psychiatric comorbidity. Journal of Psychiatric Research, 45, 1264-8. 